\title{
The Contrast and Evaluation of the Biological Effects on Glucose and Lipid Metabolism by the Extract from Wolfberry Fruit and Leaf
}

\author{
Lu Ping \\ School of Life Science, Tsinghua University, Beijing, China
}

Email address:

ping115@mails.tsinghua.edu.cn

To cite this article:

Lu Ping. The Contrast and Evaluation of the Biological Effects on Glucose and Lipid Metabolism by the Extract from Wolfberry Fruit and Leaf. Science Discovery. Vol. 4, No. 5, 2016, pp. 275-279. doi: 10.11648/j.sd.20160405.12

Received: July 16, 2016; Accepted: August 15, 2016; Published: August 25, 2016

\begin{abstract}
This study is aimed to clarify the mechanism of wolfberry fruit and leaf extract's regulation of lipid metabolism, and to provide the necessary theoretical basis for the possible uses of wolfberry fruit extract as drugs improving lipid metabolism. We have investigated the effects of wolfberry extract on the proliferation and differentiation as well as lipid metabolism of preadipocytes on cell level, and have evaluated the influences of wolfberry extract on obese mice body weight, oral glucose tolerance and blood glucose. The comparison between the effects of different parts of wolfberry and the combination of experiments of cell and animal level are done for the first time. Results show that: neither wolfberry fruit nor wolfberry leaf has toxicity on 3T3-L1 preadipocytes; wolfberry extract can promote adipocyte differentiation and intracellular lipid accumulation, while wolfberry leaf does not have such effects; fruit extract can improve the $C / E B P \alpha, P P A R-\gamma$ gene expression levels and activate the transcriptional activity of $P P A R-\gamma$, while leaf does not have such effects; both wolfberry fruit and wolfberry leaf can reduce the blood glucose and improve the oral glucose tolerance of obese mice, and wolfberry fruit has a stronger effect, but in the meanwhile they also increase the mice's body weight. Based on the results above, we can conclude that wolfberry fruit extract ingredients can regulate glucose and lipid metabolism to some extent. Thus, it has the potential to work as a kind of medicine to improve lipid metabolism and to cure type 2 diabetes.
\end{abstract}

Keywords: Wolfberry Extract, 3T3-L1 Preadipocytes, Adipocyte Differentiation, Glucose and Lipid Metabolism, PPAR- $\gamma$

\section{枸杞不同部位提取物调节糖脂代谢的生物学效应对比及评价}

\section{平陆}

生命学院, 清华大学, 北京, 中国

\section{邮箱}

ping115@mails. tsinghua. edu.cn

摘要: 枸杞是一种传统中药材。现代研究表明, 枸杞子与叶具有降血糖、降血脂、保肝、抗衰老、抗肿瘤的作用。本 文旨在阐明枸杞果实与叶提取物调节脂质代谢的作用机制, 将为枸杞提取物作为改善脂代谢紊乱药物提供必要的理论 依据。研究了枸杞提取物对前脂肪细胞增殖分化及糖脂代谢的影响; 在动物水平上, 评价枸杞提取物对肥胖小鼠体重, 口服糖耐量及血糖的影响。首次结合细胞与动物层面, 比较枸杞不同部位提取物的影响。结果显示, 枸杞子、枸杞叶 提取物对 $3 \mathrm{~T} 3-\mathrm{L} 1$ 前脂肪细胞均无毒性; 枸杞子提取物可促进前脂肪细胞分化及胞内脂质积累, 提高 $C / E B P a 、 P P A R-\gamma$ 的基因表达水平, 激活 $P P A R-\gamma$ 的转录活性, 枸杞叶提取物没有相应作用; 枸杞子与枸杞叶提取物均可降低肥胖小鼠的 血糖, 改善口服糖耐量, 但会使小鼠略微增重, 枸杞子提取物的效果优于枸杞叶提取物。以上结果表明, 枸杞子提取 物可在一定程度上调节糖脂代谢, 具有成为调节脂代谢与治疗糖尿病药物的可能。 
关键词: 枸杞提取物, 3T3L1前脂肪细胞, 脂肪细胞分化, 糖脂代谢, PPAR- $\gamma$

\section{1. 引言}

\section{1 . 前言}

枸杞是一种传统中药材, 其叶、花、果实、根均可入 药。明李时珍《本草纲目》记载: “春采枸杞叶, 名天精 草; 夏采花, 名长生草; 秋采子, 名枸杞子; 冬采根, 名 地骨皮”。现代研究表明, 枸杞具有降血糖、降血脂、保 肝、抗衰老、抗肿瘤的作用 [1]。此项课题研究会将枸杞 不同部位提取物的影响细胞脂代谢的能力进行比较, 研究 其调节糖尿病脂代谢紊乱的功效。

糖尿病是一组由于胰岛素分泌缺陷和/或胰岛素作用 障碍所致的以高血糖为特征的代谢性疾病。持续高血糖与 长期代谢紊乱等可导致全身组织器官, 特别是眼、肾、心 血管及神经系统的损害及其功能障碍和衰竭, 而脂代谢紊 乱是糖尿病并发心血管疾病的首要危险因子之一。因此, 对于药物调节脂代谢紊乱的研究有重要意义。

\section{2. 枸杞}

\subsection{1. 枸杞概述}

枸杞 (Lycium Chinense) 是茄科枸杞属的多分枝灌 木植物, 高0.5-1米, 栽培时可达2米多。全世界枸杞属植 物约有 80 种, 多数种分布在以美国亚利桑那州和阿根廷为 中心的南北美洲。枸杞在中国主要有宁夏、甘肃、青海、 新疆等产地。

\subsection{2. 枸杞子提取物及其功效}

枸杞子为茄科植物宁夏枸杞 (Lycium barbarum L. ) 的干燥成熟果实, 是一味传统常用中药, 《本草纲目》谓 其补肾、润肺、生精、益气。枸杞子性平、味甘, 具有滋 补肝肾, 益精明目的功效。

宁夏枸杞的化学成分主要含枸杞多糖 [2] (LBP)、甜菜 碱 [3] (Betaine)、类胡萝卜素及类胡萝卜素酯 $[4]$ 、维生 素C [5]、莨宕亭 $[6]$ (scopoletin)、多种氨基酸及微量元 素K、Na、Ca、Mg、Cu、Fe、Mn、Zn、P等。此外, 尚从枸 杞中分得枸杞素A-D、脑式脂类、玉蜀㯟黄素及玉蜀㯟黄 素二棕㭣酸等。

据考察研究, 枸杞子提取物具有抗化学性肝损伤 [7]、 抗衰老 [8]、扩张血管 [9]、抑菌抗炎 [10]、提高免疫力和 延长寿命 [11]等功效。也有研究表明, 枸杞多糖具有降低 血糖 [12]、降低血脂的功效且存在量效关系 [13]。

\subsection{3. 枸杞叶提取物及其功效}

枸杞的嫩茎叶统称枸杞叶, 俗称天精草、地仙苗。中 医学认为, 枸杞叶性凉、味甘、微苦, 具有除烦益志、坚筋 耐老、补虚益精、养肝明目、除热毒、散疮肿、补五劳七 伤、去皮肤骨节间风等功效。
枸杞叶的活性成分主要含水、脂肪、蛋白质、碳水化 合物、纤维素、钙、磷、铁、胡夢卜素、硫胺素、核黄素、 尼克酸、抗坏血酸 $[14]$ 、黄酮类化合物 [15]等。

枸杞叶的提取物具有降血糖 [16]、降血脂、抗肌肉疲 劳 [17]等的功效。

\section{3. 糖尿病}

\subsection{1. 糖尿病概述}

糖尿病是一组胰岛素分泌缺陷或胰岛素功能障碍所 致的以高血糖为特征的代谢性疾病。持续高血糖与长期代 谢紊乱等可导致全身组织器官, 特别是眼、肾、心血管及 神经系统的损害及其功能障碍和衰竭。严重者可引起失水, 电解质紊乱和酸碱平衡失调等急性并发症酮症酸中毒和 高渗昏迷。

\section{3. 2. I 型糖尿病与 II 型糖尿病}

I 型糖尿病患者有胰岛 $\beta$ 细胞破坏, 引起胰岛素绝对 缺乏，有酮症酸中毒倾向。可发生于任何年龄，但多见于 青少年。起病急。代谢紊乱症状明显, 患者需注射胰岛素 以维持生命。包括免疫介导和特发性两种亚型。免疫介导 糖尿病常有一种或多种自身抗体存在, 例如胰岛细胞抗体 (ICA)、胰岛素自身抗体 (IAA) 和谷氨酸脱羧酶65(GAD65) 抗体等。II 型糖尿病患者大部分超重或肥胖, 也可发生于 任何年龄, 但多见于成年人。以胰岛素抵抗、胰岛素分泌 不足为主要病因。患者在疾病初期大多不需要胰岛素治疗。 通常无酮症酸中毒倾向, 但在感染等应激情况下, 也可诱 发酮症酸中毒。II 型糖尿病的遗传易感性较 I 型糖尿病强 烈。

\subsection{3. 糖尿病与脂代谢紊乱}

糖尿病合并脂代谢紊乱极为常见, 高脂血症是糖尿病 患者常见的并发症, 是导致脂肪肝、动脉粥样硬化等的重 要危险因素, 严重威胁人们的健康和生命。脂代谢异常是 II 型糖尿病及其并发症的原发性病理生理过程 [18], 因此 糖尿病可称为糖脂病 (Diabetesm ellipidus)。

\section{4. $P P A R-\gamma$ 与脂肪细胞分化及脂代谢}

过氧化物酶体增殖体激活受体 (PPAR) 有 $\alpha 、 \beta 、 \gamma 3$ 种类型, 可与类维生素A受体 (RXR) 结合形成异二聚体, 再 结合于特异性DNA序列 (此序列称为过氧化体增殖反应元 件即PREE), 调控靶基因的转录。 $\alpha$ 主要与脂代谢有关; $\beta$ 分布广泛, 作用尚不清楚; $\gamma$ 具有多种生物效应, 在脂肪细 胞分化、糖、脂代谢、泡沫细胞动脉粥样硬化形成、炎性 反应中起重要作用 $[19]$ 。

研究表明, $P P A R-\gamma$ 对脂肪细胞的增殖与分化均有影 响, $P P A R-\gamma$ 基因表达量的下降, 可使鸡脂肪细胞增值能 力加强, 分化能力减弱 [20], 可见, $P P A R-\gamma$ 对脂肪细胞 的分化有促进作用。 
$P P A R-\gamma$ 在脂代谢过程中起着重要作用 [21], 通过参 与脂代谢相关基因的转录调控, 从而参与脂代谢调控。

\section{2. 实验及结果}

\section{1. 细胞实验}

\subsection{1. 未分化细胞的培养}

采用小鼠3T3-L1 前脂肪细胞株, 用含 $10 \%$ 胎牛血清 (FBS) 的高糖DMEM培养基, 在 $37^{\circ} \mathrm{C} 、 5 \% \mathrm{CO}_{2}$ 的培养箱中培 养, $3 \mathrm{~d}$ 换一次液, 并按常规实验方法进行细胞传代, 细胞冻 存和复苏。在倒置光学显微镜下观察细胞形态。

\section{1. 2. 细胞增殖检测}

取对数生长期的 $3 \mathrm{~T} 3-\mathrm{L} 1$ 前脂肪细胞传代培养, 按 $5 \mathrm{X}$ 的密度接种到 96 孔板中, 待细胞融合至 $60 \%-70 \%$ 后加药, 分为空白对照组、41^1：”组 (100、50、25、12.5Mmol/L)、 枸杞子提取物组 (100、50、25、12.5Mmol/L), 枸杞叶提 取物组 (100、50、25、12.5Mmol/L), 继续培养44h后, 每 孔加 $20 \mathrm{uL} 5 \mathrm{mg} / \mathrm{mL}$ 四甲基偶氮盐即MTT, 孵育 $4 \mathrm{~h}$, 移除培养 液, 每孔加 $150 \mu 1$ 甲基亚砜, 震荡混匀, 酶标仪上测 $\mathrm{A} 492 \mathrm{~nm}$ 处吸光值。

\subsubsection{MTT试验结果及分析}

相比对照组 1 , 混有不同浓度枸杞不同部位提取物的 生长培养基培养的细胞活力均有所提高, 可见枸杞不同部 位提取物均会促进前脂肪细胞的增殖。 $0.1 \mathrm{mg} / \mathrm{ml}$ 的枸杞子、 枸杞叶提取物对细胞活力的促进最明显（图1）。

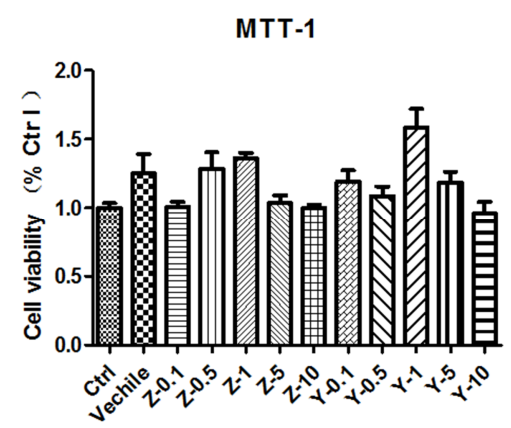

图1 培养基中加入不同浓度枸杞子、叶提取物后培养的细胞活力。横轴 为纯生长培养基 (对照组1）、纯分化培养基（对照组 2）、和混有不同 浓度枸杞不同部位提取物的生长培养基（实验组）。Ctr1代表纯生长培 养基, Vechile代表纯分化培养基, Z 代表枸杞子, Y代表枸杞叶, 浓度 量纲为 $\mathrm{mg} / \mathrm{m} 1$ 。纵轴代表细胞活力与对照组 1 的比值。

\subsection{4. 细胞分化的诱导培养}

培养基: Day 0 MDI induction media (10\% FBS/DMEM 含 IBMX $0.5 \mathrm{mM}$, Dex $1 \mu \mathrm{M}$, Insulin $10 \mu \mathrm{g} / \mathrm{ml}$ ); Day2 Insulin Media (10\% FBS/DMEM 含 Insulin $10 \mathrm{\mu g} / \mathrm{ml}$ ); Day 4 10\% FBS/DMEM。按上述时间和配方更换培养基。

\subsection{5. 油红染色}

24孔板, 分为生长培养基对照组; 分化培养基对照组; 枸杞子/叶提取物各 $0.5 \mathrm{mg} / \mathrm{ml} 、 1 \mathrm{mg} / \mathrm{ml} 、 5 \mathrm{mg} / \mathrm{ml}$ 三个浓度。

\subsection{6. 油红染色试验结果及分析}

相比分化培养基对照组, 混有枸杞叶提取物的分化培 养基培养的细胞油红染色比率低于纯分化培养基培养的 细胞, 可见枸杞叶提取物对 $3 \mathrm{t} 311$ 前脂肪细胞分化的促进 作用不明显，而枸杞子提取物对 $3 \mathrm{t} 311$ 前脂肪细胞的分化 明显起促进作用，使细胞的染色比率高于对照组。 $0.5 \mathrm{mg} / \mathrm{ml} 、 1 \mathrm{mg} / \mathrm{ml} 、 5 \mathrm{mg} / \mathrm{ml}$ 三种浓度中，促进作用随浓度 的提高而愈发明显。

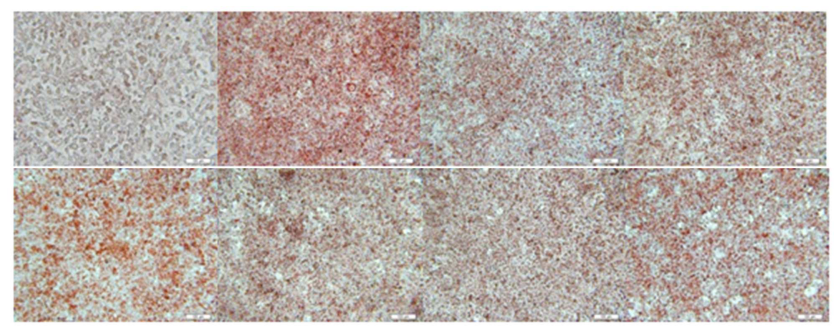

图2 油红染色镜下照片。左至右, 上至下依次为纯生长培养基, 纯分化 培养基, $0.5 \mathrm{mg} / \mathrm{ml}$ 枸杞子提取物, $1 \mathrm{mg} / \mathrm{m} 1$ 枸杞子提取物, $5 \mathrm{mg} / \mathrm{m} 1$ 枸杞子 提取物, $0.5 \mathrm{mg} / \mathrm{m} 1$ 枸杞叶提取物, $1 \mathrm{mg} / \mathrm{m} 1$ 枸杞叶提取物, $5 \mathrm{mg} / \mathrm{m} 1$ 枸杞叶 提取物。

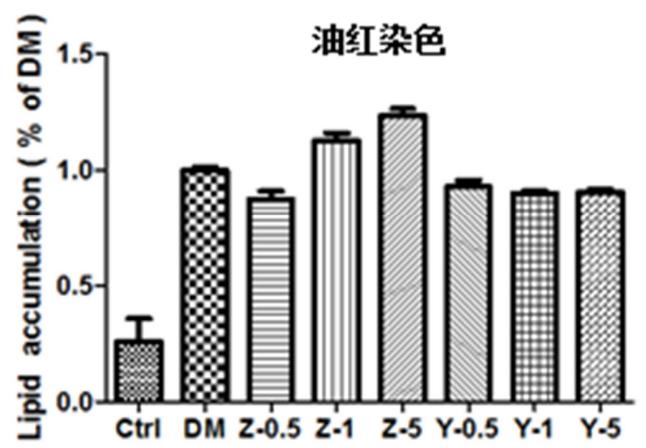

图3 培养基中加入不同浓度枸杞子、叶提取物后脂肪的积累。横轴为纯 生长培养基（对照组1）、纯分化培养基（对照组2）、和混有不同浓度 枸杞不同部位提取物的分化培养基 (实验组)。Ctr1代表纯生长培养基, Dm代表纯分化培养基, Z代表枸杞子, Y 代表枸杞叶, 浓度量纲为 $\mathrm{mg} / \mathrm{ml}$ 。 纵轴为染色比率与对照组 2 的比值, 可体现出细胞的分化状态。

\section{2. 动物实验}

用枸杞叶/子提取液、低糖饲料、高糖饲料灌胃治疗 高脂肥胖组大鼠4周，测量其体重变化如下:

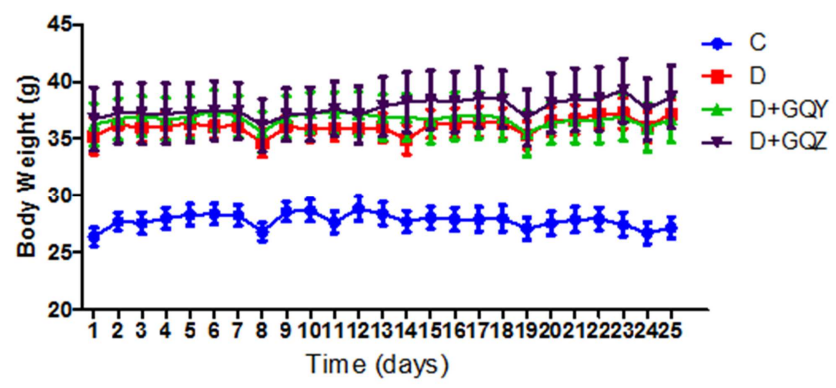

图4 大鼠体重随时间的变化。C代表对照组, D代表高脂肥胖组, D+GQZ 代表高脂肥胖组喂养枸杞子提取液, D+GQY 代表高脂肥胖组喂养枸杞叶 提取液。 
由图4可知, 高脂肥胖组服用枸杞子提取液后体重有 所上升, 服用枸杞叶提取物后体重无明显变化。

\section{3. 讨论}

已知的研究结果显示, 枸杞子作为传统的中药材, 其 组分包含核黄素、甜菜碱、胡夢卜素、硫胺素等化学成分。 其多糖组分 (Lycium barbarum polysaccharides) 作为 一种生物活性物质, 可促进免疫细胞的功能, 增强细胞因 子的合成及分泌，可依赖性地抑制肿瘤。此外，枸杞子提 取物作为天然来源的抗氧化剂, 可增强谷胱甘肽过氧化物 酶（GSH-Px）活力和超氧化物歧化酶SOD的活性, 可预防 肝脏的脂质过氧化, 保护肝脏功能。

已有研究表明, 枸杞子提取物可显著降低一型糖尿 病动物模型的血糖水平, 改善受损的糖耐量。本研究使 用的高脂饲料诱导的肥胖小鼠模型 (DI0) 可一定程度上 模拟肥胖导致的二型糖尿病脂代谢紊乱, 是国际上常用 的研究脂质代谢紊乱的疾病动物模型。实验结果显示, 枸杞果实及叶子提取物皆可降低肥胖动物模型的血糖水 平, 改善受损的葡萄糖耐量, 枸杞果实的治疗效果优于 枸杞叶提取物, 但是这两种提取物均会使小鼠体重略微 增加。

为了进一步解释枸杞果实及叶子提取物降血糖的机 制, 本文研究了枸杞提取物对前脂肪细胞的增殖及分化 过程的影响, 比较了两种提取物对前脂肪细胞分化影响 的差异。实验结果表明, 在 $0.1-5 \mathrm{mg} / \mathrm{ml}$ 的浓度范围内, 两种提取物对脂肪细胞没有明显的毒性。油红染色的结 果显示, 枸杞果实提取物可显著促进前脂肪细胞的分化, 而枸杞叶提取物对前脂肪细胞分化没有影响。同时实时 定量PCR的结果显示, 枸杞果实提取物可促进分化相关基 因PPAR $-\gamma$ 及 $C / E B P a$ 的表达, 枸杞叶提取物对这两种基 因的表达没有影响。在前脂肪细胞的分化过程中, $P P A R-\gamma$ 作为重要的转录因子调控着脂肪细胞分化的整 个过程, 枸杞果实提取物可促进前脂肪细胞的分化, 预 示枸杞果实提取物中可能含有 $P P A R-\gamma$ 的天然配体。为了 验证这可能性, 我们检测枸杞果实提取物是否能激活 PPAR $-\gamma$ 苂光素酶报告基因体系。实验结果表明枸杞果实 提取物可激活 $P P A R-\gamma$, 其提取物中含有可激活 $P P A R-\gamma$ 的活性成分。

由于本研究的提取物是一种成分复杂的混合物, 进一 步阐明枸杞果实的详细机制, 还需要分离鉴定其中的有效 活性成分。

枸杞子与叶提取物可使高脂肥胖小鼠血糖明显下降, 但与现有治疗糖尿病的药物罗格列酮相似, 枸杞子与叶提 取物会使小鼠体重增加, 其使小鼠体重增加的原因还有待 进一步探究。

再有, 虽然枸杞叶提取物对于3T3-L1前脂肪细胞的分 化能力几乎没有影响, 但是在动物实验中却有与枸杞子提 取物近似的作用效果, 可降低小鼠的空腹血糖和口服糖耐 量OGTT。因此, 无法排除枸杞叶治疗糖尿病的可能, 其降 血糖机理还有待探究。

\section{4. 结论}

本研究通过动物实验与细胞实验, 得到结论: 枸杞子、 枸杞叶提取物对 3T3-L1前脂肪细胞均无毒性。枸杞子提取 物可促进脂肪细胞的分化，提高脂肪细胞分化相关基因 $P P A R-\gamma$ 和 $C / E B P a$ 的表达。其促进作用可能是因为枸杞子 提取物可增强PPAR- $\gamma$ 的转录活性。枸杞提取物均可降低 高脂膳食诱导肥胖小鼠（DIO）的血糖水平, 改善口服糖 耐量, 枸杞子提取物的作用效果均强于枸杞叶提取物。

本研究表明, 与枸杞叶提取物相比, 枸杞子提取物更 适合 II 型糖尿病的治疗, 是一种极具发展空间的天然药物, 是一种潜在 $P P A R-\gamma$ 的天然激动剂, 可作为天然的胰岛素 增敏剂改善胰岛素抵抗。

\section{致谢}

本研究得到了中国科学院丁文军教授 张良博士的支 持。

\section{参考文献}

[1] 张云霞, 刘敦华. 枸杞功能性成分研究进展及深加工发展趋 势 [J]. 食品与药品, 2009, 11 (3) :67-69.

[2] 黄琳娟, 林颖, 田庚元, 等. 枸杞子中免疫活性成分的分离、 纯化及物理化学性质的研 究 [J]. 药学学 报, 1998, 33 (7) :512-516.

[3] 杨东辉, 王积福, 魏璐雪. 枸杞子浸亳甜菜碱的含量测定 [J]. 中国中药杂志, 1997, 22 (10) :608-610.

[4] 彭光华, 李忠, 张声华. 薄层色谱法分离鉴定枸杞子中的类 胡萝卜素 [J]. 营养学报, 1998, 20(1):76-78.

[5] 陈耀明. 枸杞子浓缩汁维生素C 含量测定法 [J]. 医学争 鸣, 1994, 15 (4) : 304-305.

[6] 李春生, 杜桂芝, 赵全成, 等. 枸杞子化学成分的研究 $[J]$. 中国中药杂志, 1990, 15(3) : 43-44.

[7] 黄琼, 黄俊明, 陈瑞仪, 等. 大豆、枸杞子、山楂复合提取物 对小鼠化学性肝损伤的保护作用 [J]. 营养学 报, 2003, 25(2) :208-211.

[8] 谢梅林, 李松林. 枸杞子提取物的抗衰老作用 [J]. 中国野生 植物资源, 1996 (2) : 9-11.

[9] 吴维明, 冯前进, 张立伟. 枸杞子提取物对大鼠离体胸主动 脉舒张作用的初步研究 [J]. 山西中医学院学 报, 2007, 8(2):18-19.

[10] 文建军. 枸杞子提取物对各种常见细菌抑菌及抗炎作用实 验研究 [J]. 医药论坛杂志, 2012, 33 (7) :23-25.

[11] 陈荣荃, 谢梅林, 钱曾年, 等. 构杞子提取物对小鼠免疫功能 和果蝇寿命的影响 [J]. 中国野生植物资源, 1995 (2) : 5-7. 
[12] 李长江, 张英锋, 高大威. 枸杞多糖分离纯化及降血糖效果 的研究 [J]. 燕山大学学报, 2014(06) :557-560.

[13] 丁园. 枸杞多糖对 2 型糖尿病大鼠血糖、血脂的影响 $[\mathrm{J}]$. 辽 宁医学院学报, 2015, 36(5) : 12-14.

[14] 齐宗韶, 李淑芳, 吴继平, 等. 枸杞子和枸杞叶化学成分的研 究——第1报枸杞子和枸杞叶的营养成分 [J]. 中国中药杂 志, 1986, $11(3): 41-43$

[15] 何微, 朱捷. 枸杞叶中总黄酮及芦丁提取工艺的研究 $[J]$. 当 代化工, 2015(11):2723-2725.

[16] 杨涓, 魏智清, 陈炜. 枸杞叶降血糖作用的初步研究 [J]. 四 川中医, 2009, 27 (4) : 47-49
[17] 黄欣, 赵海龙, 尹宏. 青海枸杞叶提取物对低氧递增游泳训 练小鼠运动能力的影响 $[\mathrm{J}]$. 辽宁中医药大学学 报, 2008, 10(7):141-143.

[18] Mcgarry J D. Banting Lecture 2001 Dysregulation of Fatty Acid Metabolism in the Etiology of Type 2 Diabetes [J]. Diabetes, 2002, 51(1):7-18.

[19] 李利平, 付方明. PPAR $\gamma$ 研究进展 $[J]$. 国际内分泌代谢杂 志, 2003, 23 (1) : 29-32.

[20] 王丽, 那威, 王宇祥, 等. 鸡PPAR $\gamma$ 基因的表达特性及其对脂 肪细胞增殖分化的影响 [J]. 遗传, 2012, 34 (4) : 454-464.

[21] Lehrke M, Lazar M A. The Many Faces of PPAR $\gamma$ [J]. Cell, 2005, $123(6): 993-999$. 